\title{
Complexation and enhancement of temozolomide solubility with cyclodextrins
}

\author{
Berna Gürten', Elçin Yenigül ${ }^{1}$, Ali Demir Sezer ${ }^{2 *}$, Seyda Malta1 \\ ${ }^{1}$ Department of Chemical Engineering, Faculty of Engineering, Yeditepe University, Ataşehir, Istanbul, Turkey, ${ }^{2}$ Department of \\ Pharmaceutical Biotechnology, Faculty of Pharmacy, Marmara University, Haydarpaşa, Istanbul, Turkey
}

\begin{abstract}
Temozolomide is a poorly soluble anti-cancer drug used in the treatment of some brain cancers. Following literature reports about the enhancement of solubility and stability for these kinds of drugs upon complexation with cyclodextrins, we aimed to form an inclusion complex between temozolomide and the different types of cyclodextrins (CDs) to enhance its solubility. In this study, three different cyclodextrins $(\beta-C D$, hydroxyl- $\beta-C D$ and $\gamma-C D)$ were used, and changes in solubility was measured by UV-Vis Spectroscopy and HPLC. Morphological changes upon complexation were shown by the Scanning Electron Microscope (SEM), and weight loss profiles with respect to temperatures which were unique to the compounds were shown by Thermogravimetric Analysis. Changes in heat release profiles were shown by Differential Scanning Calorimeter (DSC). Drug solubility was measured to be increased to around 25\% for 1:1 molar ratio for all used CD complexations. Changes of morphology, heat release and weight loss profiles are consistent with the formation of an inclusion complex between CDs and temozolomide. In this study, success was shown in the enhancement of temozolomide solubility upon complexation with different types of CDs. It has been demonstrated that cyclodextrins can be used as complexing agents for poorly soluble anti-cancer drugs, increasing their solubility and hence drug availability.
\end{abstract}

Keywords: Temozolomide/solubility/complexation. Cyclodextrins. Chromatography. Calorimetry.

\section{INTRODUCTION}

Temozolomide (TMZ) is a second generation imidazotetrazinone derivative with methylating properties. It is used as an oral cytotoxic agent for adjuvant chemotherapy in malignant primary brain tumors such as glioblastoma (Stupp et al., 2005) and malignant glioma (glioblastoma multiforme and anaplastic astrocytoma) (Brada et al., 1999; Marchesi et al., 2007). The drug contains an imidazole group connected to a tetrazole ring (Figure 1) (Khan et al., 2016). Due to its relatively high cytotoxicity for cancer cells, TMZ is commonly preferred by clinicians (Payne, Pratap, Middleton, 2005). TMZ is more of a prodrug and its chemical decomposition to 3-methyl-(triazen-1-yl) imidazole-4-carboxamide (MTIC) alkylates nucleophiles (i.e. DNA) and causes cell death (Kim et al., 2001). This process is highly $\mathrm{pH}$ dependent since TMZ exerts in vivo

\footnotetext{
*Correspondence: A. D. Sezer. Department of Pharmaceutical Biotechnology, Marmara University, Haydarpaşa 34668, Istanbul, Turkey. Tel: +90 21641429 63, Fax: +9021634529 52. E-mail: adsezer@marmara.edu.tr
}

activity by spontaneous hydroxylation at a physiologic pH (Andrasi et al., 2010).<smiles>Cn1nnc2c(C(N)=O)ncn2c1=O</smiles>

FIGURE 1 - Structure of TMZ.

TMZ brings additional benefits, so that it helps the crossing of DTIC (5-(3,3-dimethyl-1-triazenyl) imidazole-4-carboxamide) analogues through the blood brain barrier, which is another member of the alkylating agent class with a similar mechanism of action in metastatic melanoma. It is known that metastatic melanoma frequently metastasizes to the brain (Zuckerman et al., 2011). It is important to deliver an appropriate level of the active chemotherapeutic agent to the tumor area to cross the blood brain barrier. Aqueous solubility is known as one of the major concerns of conventional chemotherapy 
due to the mostly non-polar nature of chemotherapeutic agents (Chidambaram, Manavalan, Kathiresan, 2011). As a result, various delivery vehicles can be used to increase the solubility of conventional chemotherapy selectivity. Apart from solubility, the chemical stability of TMZ is also worth consideration. Once TMZ is delivered in the body, it quickly decomposes to its active agent, MTIC, at a neutral $\mathrm{pH}$. Therefore, any delivery vehicle of the drug should be prepared in the acidic solution and then lyophilized for stability.

Cyclodextrins (CDs) are molecular chelating agents belonging to a class of cyclic oligosaccharides which consist of $(\alpha-1,4)$-linked $\alpha$-D-glucopyranose units. These compounds contain a lipophilic inner cavity and a hydrophilic outer surface. CDs have significant capacities to form inclusion complexes according to their core-shell structure. As a result of molecular complexation, these molecules are appropriate hosts to be used for drug-delivery, many industrial products, technologies and analytical methods (Loftsson et al., 2002; Del Valle, 2004; Loftsson et al., 2005). Natural CD and its synthetic derivatives can be employed to enhance certain physicochemical properties of the drug such as dissolution, solubility, stability or release rates (Uekama, Otagiri, 1987). There are different derivatives of CDs, but $\beta$-CD is the most commonly used form, because it is easily produced and cheaper with respect to the others. Depending on the number of sugar units in cyclodextrins, the cavity size can be altered to accommodate different drugs. $\alpha, \beta, \gamma$ cyclodextrins refer to the sugar unit numbers of 6,7 and 8 , respectively. Apart from tailoring the cavity size, the solubility of cyclodextrins in aqueous medium can also be modified by hydroxypropyl, methyl or sulfobutyl ether to derive the structure (Loftsson et al., 2005; Uekama, Otagiri, 1987).

Solubility and stability of various host agents of pharmaceutical agents, such as protein and peptide, steroids or low molecular weight agents, have been studied and improved by using natural or synthetic kinds of CD compounds. The inclusion complexation of this host-guest systems exhibits considerable intramolecular interactions, such as hydrogen bonding, hydrophobic interactions or electrostatic attractions (Uekama, Otagiri, 1987; Aiassa et al., 2015). The formation of an inclusion complex between TMZ and $\beta-C D$ is schematically shown in Figure 2.

Generally, drugs should be sufficiently hydrophobic in order to penetrate biological membranes via passive diffusion, but at the same time possess some hydrophilicity in aqueous medium. For many pharmaceutical applications, it was shown that inclusion complex helped to improve solubility (Aiassa et al., 2015; Atipairin, Sawatdee, 2016;

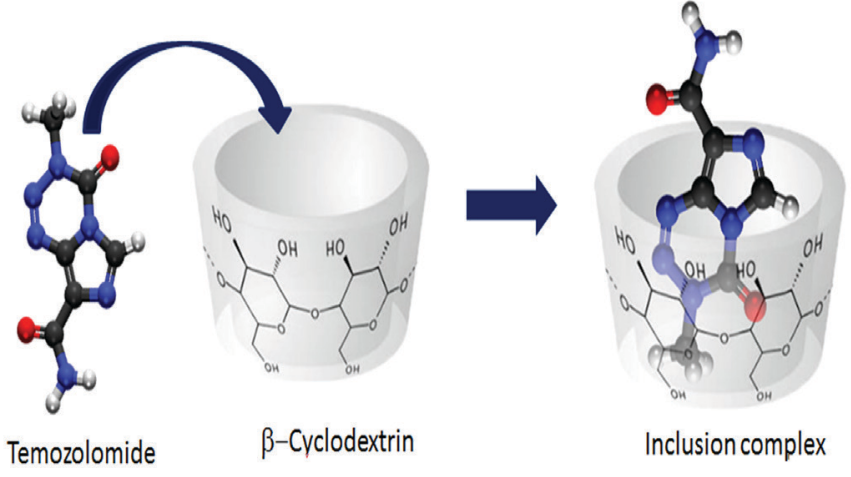

FIGURE 2 - Inclusion complex between TMZ and $\beta$-cyclodextrin.

Freitas et al., 2012; Ol'khovich et al., 2014; Priya et al., 2013; Shende et al., 2015; Zhang et al., 2013a; Zhang et al., 2013b; Franco et al., 2009; Ruan et al., 2005; Tang et al., 2015; De Araujo et al., 2008), stability (Freitas et al., 2012; Shende et al., 2015; Kicuntod et al., 2016), bioavailability (Atipairin, Sawatdee, 2016; Zhang et al., 2013a), and therapeutic effect (Zhang et al., 2013b), between various drugs and cyclodextrins. Inclusion complexes of epothilone (Xiao et al., 2014), oxaliplatin (Zhang et al., 2016), paclitaxel (Hamada et al., 2006), etc. were found to be effective for anti-tumor therapy.

The size of the hydrophobic cavity depends on the number of sugar units; hence solubility depends on derivation of the cyclodextrin where both may play a role in the optimization of complex formation. In the present study, three different cyclodextrins $(\beta$-cyclodextrin ( $\beta$-CD), 2-hydroxy propyl- $\beta$-cyclodextrin (H- $\beta-C D), 2-$ hydroxypropyl- $\gamma$-cyclodextrin $(\mathrm{H}-\gamma-\mathrm{CD})$ were employed to optimize the complex formation. The solubility of TMZ in water is quite low. Enhancing its solubility would be a great achievement. In the present study, we aimed to enhance the solubility of TMZ by using different kinds of cyclodextrins. We employed several techniques to show that an inclusion complex between the drug and CDs were formed, which was responsible for solubility enhancement.

\section{MATERIAL AND METHODS}

\section{Material}

TMZ (99.38\%) was donated by Kocak Farma, Istanbul, Turkey. Hydrochloric acid (37\%, Sigma Aldrich), acetic acid ( $\geq 99.8 \%$, Sigma Aldrich), and methanol (99.7\%, Sigma Aldrich) were also received and used without any chemical procedures. $\beta$-cyclodextrin $(\beta-C D)$, hydroxypropyl- $\beta$-cyclodextrin $(\mathrm{H}-\beta-\mathrm{CD})$, and hydroxypropyl- $\gamma$ cyclodextrin $(\mathrm{H}-\gamma \mathrm{CD})$ were purchased 
from Sigma Aldrich. The water used was distilled by reverse osmosis using GFL 2004.

\section{Determination of the solubility of TMZ}

Saturation of TMZ concentration was determined by adding an arbitrary amount of excess TMZ in $5 \mathrm{~mL}$ $0.1 \mathrm{M} \mathrm{HCl}$. After vortexing, followed by sonication for 2 minutes, the excess TMZ was removed by centrifugation. The amount of TMZ in the supernatant was determined by UV-Vis Spectroscopy (BioSpec-1601 Shimadzu, Japan), measuring absorbance at $330 \mathrm{~nm}$. Once this concentration was determined, TMZ solutions of lower concentrations were prepared by dissolving different amounts of the drug again in $5 \mathrm{~mL} 0.1 \mathrm{M} \mathrm{HCl}$ and measuring their absorbance values. $\mathrm{HCl}$ was used as a solvent to prevent decomposition of the drug into its active agent at neutral pH (Babu, Sanphui, Nangia, 2012).

\section{Preparation of TMZ-cyclodextrin inclusion complex solutions}

The complexes of TMZ were formed with three different cyclodextrins $\beta$-cyclodextrin $(\beta$ $\mathrm{CD})$, hydroxypropyl- $\beta$-cyclodextrin $(\mathrm{H}-\beta-\mathrm{CD})$, and hydroxypropyl- $\gamma$-cyclodextrin $(\mathrm{H}-\gamma-\mathrm{CD})$ that contain six to eight membered rings. TMZ:CD solutions were prepared at different molar ratios as 1:1, 1:2 and 1:5.

Briefly, the required amount of cyclodextrin was first weighed and a $0.1 \mathrm{M} \mathrm{HCl}$ solution was added to obtain a total amount of $0.5 \mathrm{~g}$ solution. An excess amount of TMZ (7.25 mg, i.e. approximately two times higher than its solubility) was weighed separately and the cyclodextrin solution $(0.5 \mathrm{~g})$ was added to the weighed $\mathrm{TMZ}$ and the resulting solution was vortexed. After vortexing, $0.1 \mathrm{M} \mathrm{HCl}$ was added to the solution in order to obtain a $1.0 \mathrm{~g}$ solution. This solution was then shaken at $250 \mathrm{rpm}$ at 37 for one hour using an orbital shaker (LABWIT, ZWYR-240, Ashwood, Australian), followed by centrifugation (Hettich, EBA 21, Beverly, USA) at $6000 \mathrm{rpm}$ for 5 minutes. The supernatant was freeze-dried (SCANVAC, cool-safe, Lynge, Denmark) to obtain the TMZ-CD complex. The same procedure was applied for all cyclodextrins (Del Valle, 2004; Loftsson et al., 2005). The preparation process of TMZ-CD inclusion complex is schematically shown in Figure 3.

\section{Preparation of TMZ-cyclodextrin physical mixtures}

Excess amount of TMZ (7.25 mg) was mixed with $26.5 \mathrm{mg} \beta-\mathrm{CD}$ (1:5 mol ratio) in the solid phase using a spatula and kept at $4{ }^{\circ} \mathrm{C}$ until measurements. SEM, DSC and TGA measurements were all carried out in the solid phase.

\section{Complex formation of TMZ-CD}

Scanning electron microscopy (SEM, JEOL Ltd., JSM-5910LV, Akishima, Japan) was used to observe the morphologies of TMZ, CD, TMZ-CD complex and the TMZ-CD physical mixture. The difference

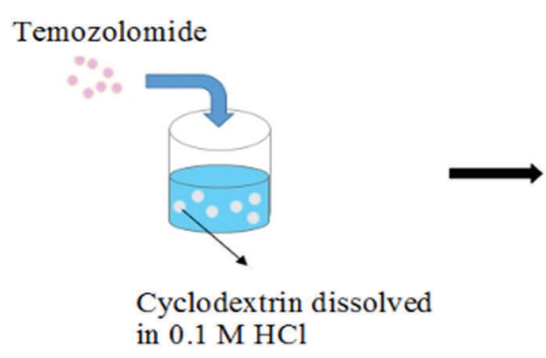

Dry TMZ:CD inclusion complex particles
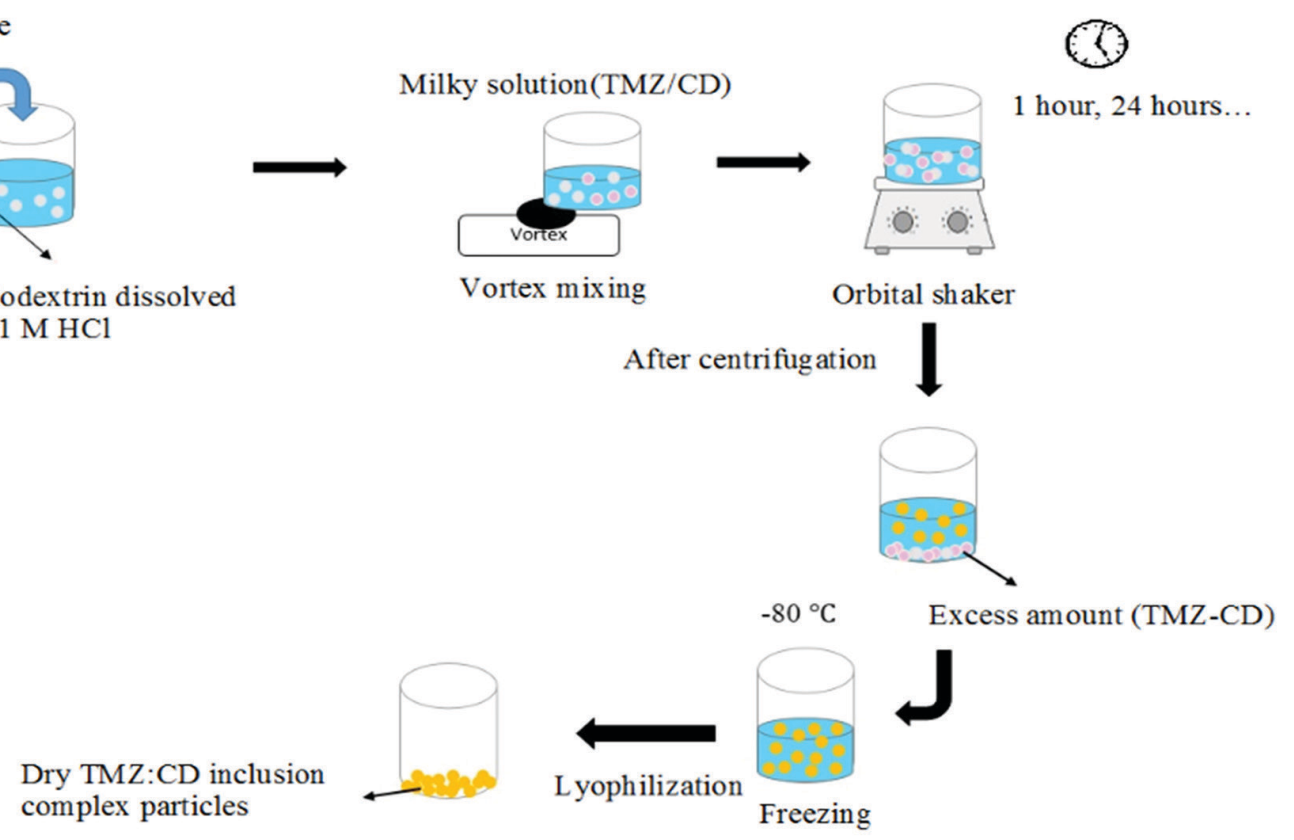

FIGURE 3 - Preparation process of TMZ-CD inclusion complex. 
in morphology of the TMZ-CD complex and TMZCD physical mixture is interpreted as a support for the formation of the complex. DSC (Setaram instrumentation, DSC 131, Caluire, France) was used to compare the endothermic and exothermic peaks of TMZ, CD, TMZ-CD complex and TMZ-CD physical mixture. In the physical mixture, the TMZ and CD peaks are expected to remain in the original positions of TMZ and $\mathrm{CD}$, whereas upon complexation new peaks are expected to appear, indicating the formation of a new entity. A thermogravimetric analysis (Perkin Elmer, Pyris 1 TGA, Beacousfield, England) was performed for the same systems. Again, upon complexation the baseline temperatures are expected to change, whereas for the physical mixture, the baseline temperatures should be identical with the CD and TMZ.

\section{Enhancement of solubility of TMZ}

In order to investigate if TMZ-CD complexation enhances solubility of TMZ, after mixing, the solution was shaken at $300 \mathrm{rpm}$ for 24 hours at $37^{\circ} \mathrm{C}$. The undissolved TMZ was removed by centrifuging at $6000 \mathrm{rpm}$ for 5 minutes. The supernatant was then diluted 200 times with $0.1 \mathrm{M} \mathrm{HCl}$ to measure the amount of solubilized TMZ using UV-Vis Spectrophotometer in the 200-450 nm range following TMZ max absorbance value at $330 \mathrm{~nm}$ and HPLC. The HPLC system consists of 1525 Binary
Pump, 2487 UV-Vis Detector, 717 Autosampler and a thermal column manager unit. A computer was also used to process raw data. In the HPLC reverse phase $\mathrm{C} 18$ column (X-Bridge $150 \mathrm{~mm} \times 0.6 \mathrm{~cm}, 0.5 \mu \mathrm{m}$, Waters was used. Water containing $0.5 \%$ acetic acid / methanol $(80: 20 \mathrm{v} / \mathrm{v})$ was prepared for the mobile phase. A standard calibration curve was obtained with five different TMZ concentrations $\left(\mathrm{R}^{2}=0.998\right)$ and three samples were used, and each sample from three injections was measured.

\section{RESULTS}

\section{Solubility curve}

As the concentration of TMZ increases, its absorbance at $200 \mathrm{~nm}$ increases linearly up to a critical concentration above which the absorbance value remains constant, regardless of increase in the amount of TMZ in the solution. This critical concentration gives the solubility value of TMZ in water at room temperature. By measuring absorbance values at $330 \mathrm{~nm}$, the Temozolomide solubility was determined as $3.46 \mathrm{mg} / \mathrm{mL}$ (Figure 4).

\section{Complex formation}

In order to show that a complex was formed between the TMZ and CDs, different techniques were employed. In all measurements, TMZ, CD, TMZ-CD physical mixture

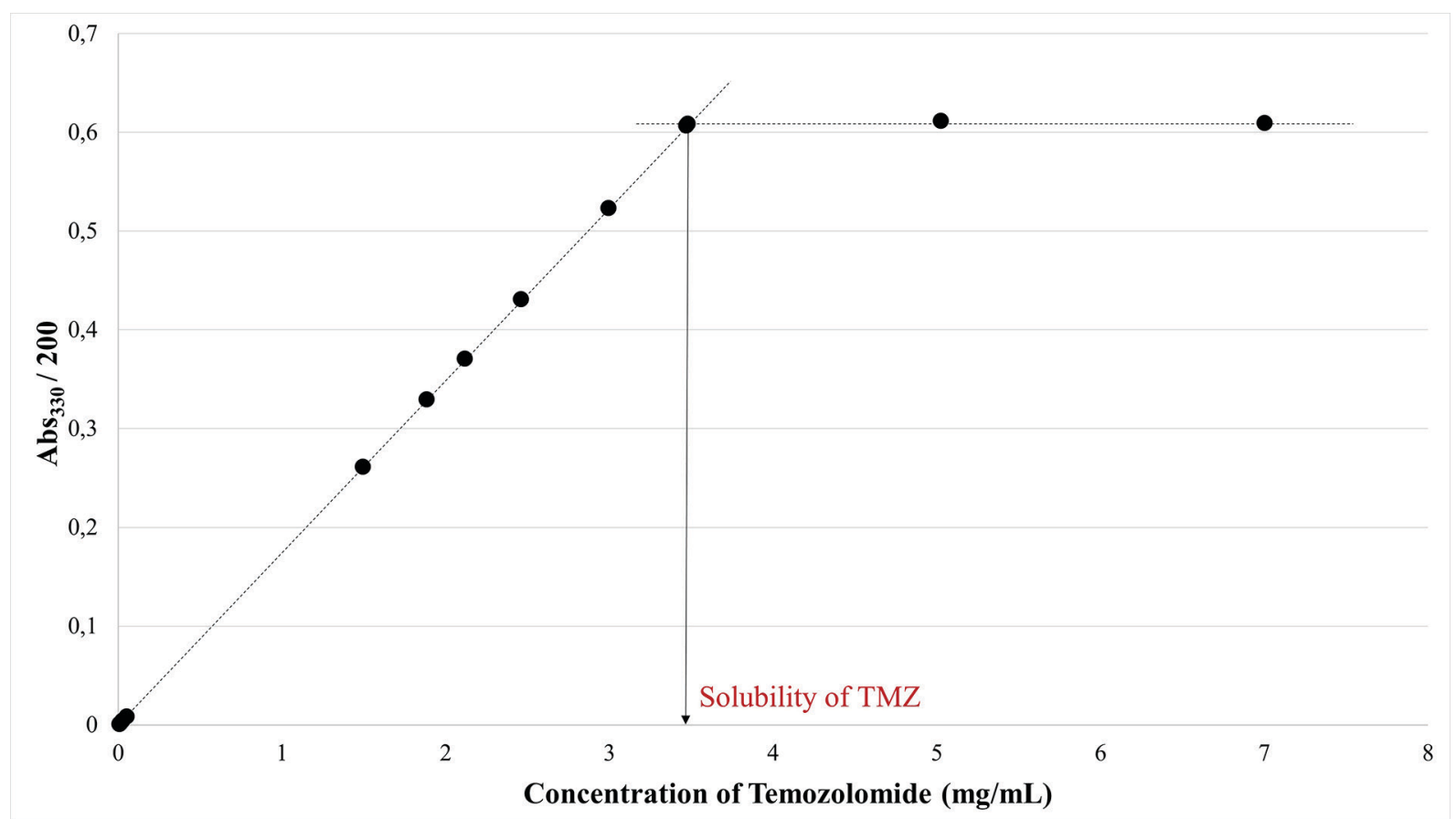

FIGURE 4 - Absorbance of TMZ at $330 \mathrm{~nm}$ for different concentrations where the minimum concentration for maximum absorbance denotes the solubility of TMZ. 
(1:1) and TMZ-CD complex (1:1) were compared. SEM was used to show clear morphology changes which occurred upon complex formation. In Figure $5 \mathrm{a}$ and $\mathrm{b}$, the TMZ and $\beta-C D$ morphologies are observed. In the physical mixture (Figure 5c), the individual TMZ and $\beta$-CD morphologies could be identified. Upon formation of a complex, a new morphology was observed where neither TMZ nor $\beta$-CD could be distinguished.

Another technique employed was TGA. TGA showed the weight loss of materials upon heating. Weight loss occurs due to decomposition of material, and the baseline temperature of this decomposition was particular for the compound. As a result, it can be used as a verification tool for that compound.

In Figure 6, the dark blue line shows the weight loss profile of TMZ, with a baseline temperature of $229^{\circ} \mathrm{C}$. The green line is the weight loss profile of $\beta-C D$, with a baseline temperature of $353{ }^{\circ} \mathrm{C}$.

The initial weight loss observed until about $100^{\circ} \mathrm{C}$ is due to the water loss in the structure (Zhang et al., 2013b; Trotta, Zanetti, Camino, 2000). In the physical mixture (yellow line), two baseline temperatures were observed, one can be associated with TMZ $\left(227^{\circ} \mathrm{C}\right)$ and the other with $\beta-C D\left(351^{\circ} \mathrm{C}\right)$. The baseline values were very similar to those of pure TMZ and $\beta-\mathrm{CD}$, which was expected from the physical mixture. In the complex, two baseline temperatures from TMZ and $\beta-C D$ were observed, with temperature shifts of 7 and $15{ }^{\circ} \mathrm{C}$, respectively.
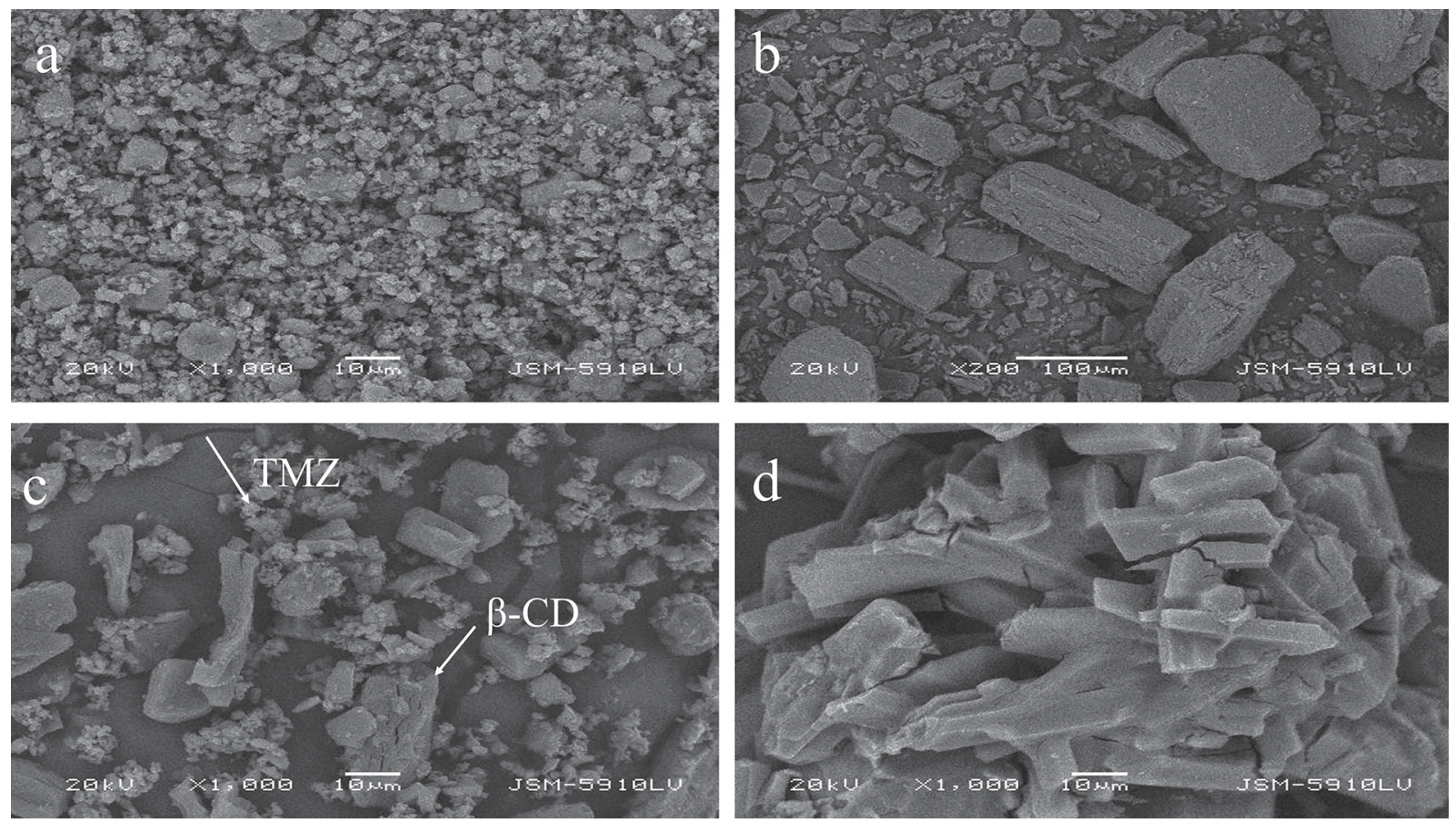

FIGURE 5 - SEM images of (a) TMZ, (b) $\beta$-CD, (c) TMZ and $\beta$-CD physical mixture where free TMZ and $\beta$-CD are clearly marked with arrows (d) TMZ and $\beta-C D$ inclusion complex where a new structure without free TMZ is revealed. 


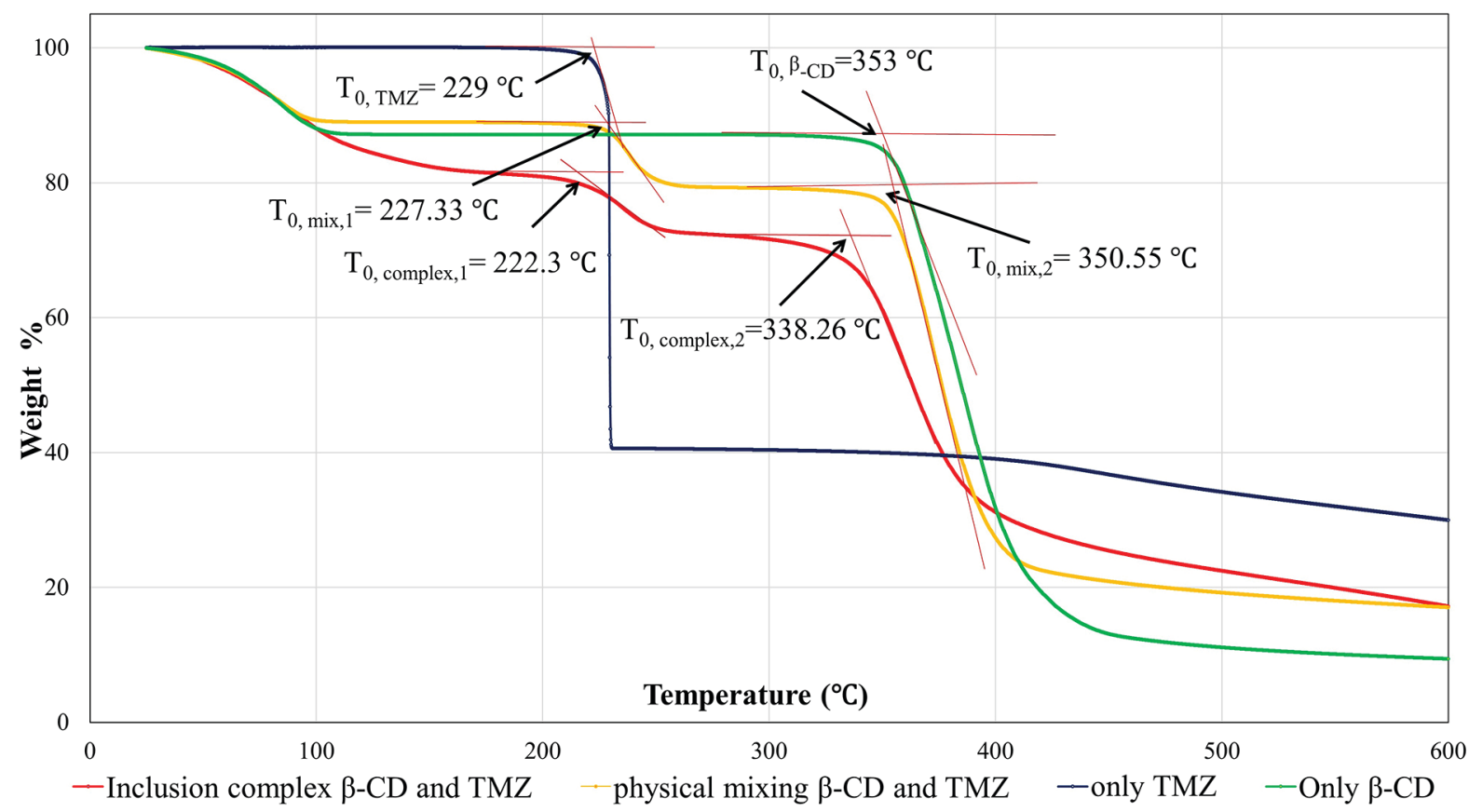

FIGURE 6 - TGA curves of TMZ- $\beta-C D$ inclusion complex and physical mixing.

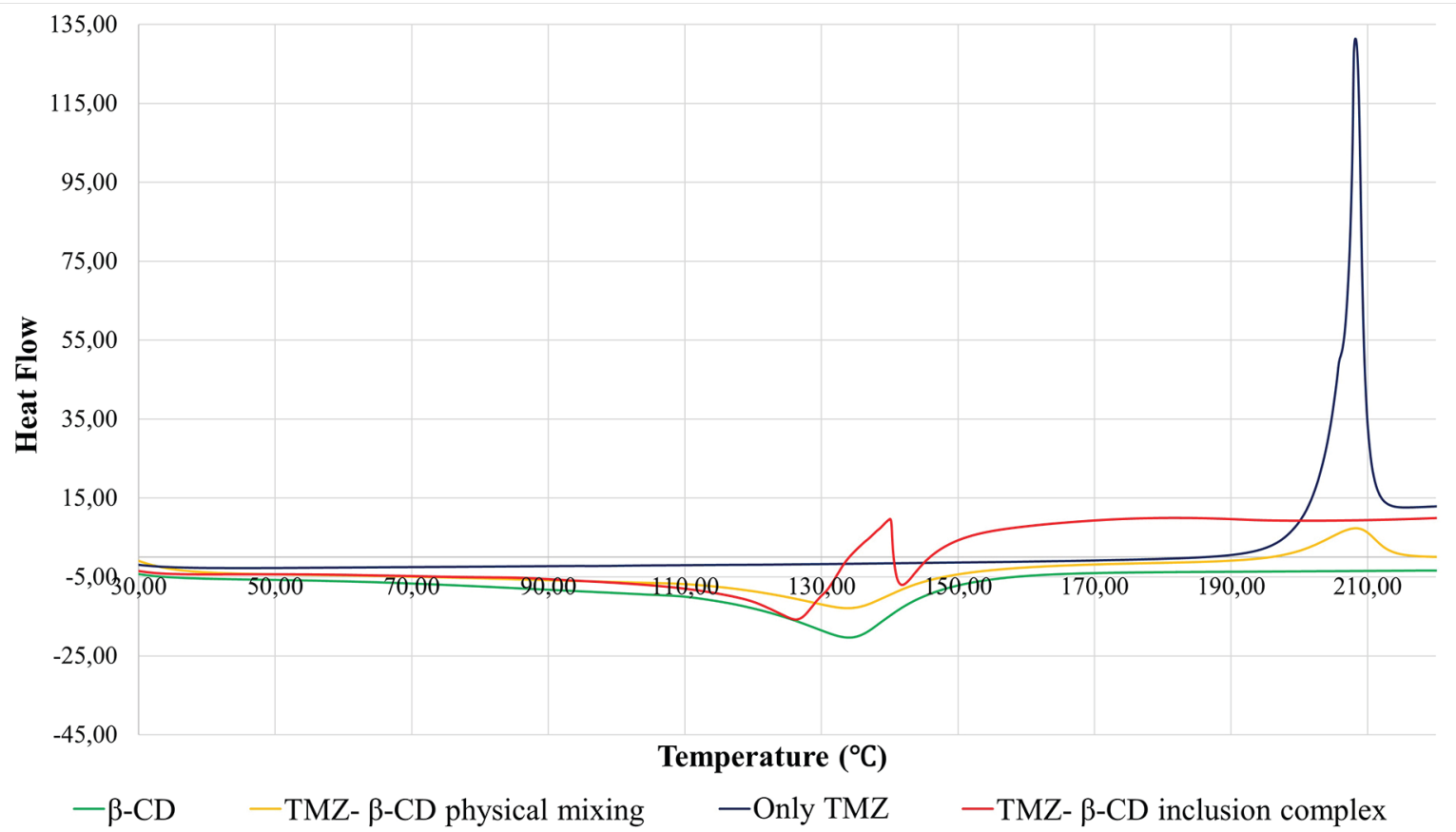

FIGURE 7 - DSC curves of TMZ- $\beta-C D$ inclusion complex and physical mixing.

amount of solubilized TMZ in all CD mixtures is shown in Table I.

In Figure 8, it was clearly showed that the amount of solubilized TMZ was higher in the presence of CDs regardless of the type of CD used.

In order to more accurately measure the concentration of solubilized TMZ in the presence of different CDs as well as in their different mixing ratios after one hour and 24 hours, the HPLC method was used. The results are demonstrated in Table II which shows that the amount of TMZ solubilized does not change from one hour to 24 hours.

The solubilized amount of TMZ in the presence of different CDs after one hour is plotted in Figure 9. 
TABLE I - Amount of solubilized TMZ in $\mathrm{mg} / \mathrm{mL}$ in $0.1 \mathrm{M} \mathrm{HCl}$ after the formation of inclusion complex after 24 hours

\begin{tabular}{lcc}
\hline Formulations & $\begin{array}{c}\text { 1 mol TMZ: 1 mol } \\
\text { CD (mg/mL) }\end{array}$ & \% Enhancement \\
\hline Only TMZ & $3.46 \pm 0.07$ & \\
$\beta$-CD-TMZ & $4.34 \pm 0.06$ & 25 \\
Hydroxy- $\beta$-CD- & $4.97 \pm 0.12$ & 43 \\
TMZ & & \\
Hydroxy- $\gamma$-CD- & $4.32 \pm 0.06$ & 25 \\
TMZ & & \\
\hline
\end{tabular}

Solubility enhancements of about $25 \%$ are obtained by using different types of CDs. These results showed that TMZ solubility increased as the CD amount in the mixing ratio increased; however, this increase was not found to be proportional to the added $\mathrm{CD}$. It is suggested that the $1: 1$ mixing ratio was sufficient to be used in this system and an enhancement in solubility of about $25 \%$ could be achieved.

\section{DISCUSSION}

Solubility and hence bioavailability of temozolomide have difficulties of enhancement, as a result no studies have been performed to in the literature. In the present study, it is suggested that cyclodextrins may be good agents to help resolve chemical issues through complexation of the drug. Literature studies have indicated that hydrophobic drugs could form inclusion complexes with cyclodextrins (Freitas et al., 2012; Ol'khovich et al., 2014). In line with the literature, three cyclodextrins used in the present study with different hydrophilicities and different hydrophobic cavity sizes optimized complexation with temozolomide (Xiao et al., 2014; Zhang et al., 2016; Hamada et al., 2006). In order to ensure that there is indeed complexation and not just a physical mixture between temozolomide and CDs, the morphological differences between physical mixture and the product obtained using SEM, along with differences in weight loss and heat loss profiles, using TGA and DSC, respectively were investigated. In the SEM images in Figure 5, both $\mathrm{TMZ}$ and $\mathrm{CD}$ are clearly distinguished in the physical mixture (Figure 5c), whereas a new morphology is observed for the complex (Figure 5d). These results are similar to those obtained by Maestrelli et al. (2010) where upon the formation of an inclusion complex, neither the drug nor the CD is distinguished in the SEM images, but a new morphology is observed.

The TGA data shown in Figure 6 clearly indicate the difference between the physical mixture and the complex. In the physical mixture, the baseline temperatures for weight loss are very similar to those of the constituents alone, whereas in the complex a new weight loss profile is clearly visible. In the physical mixture, baseline temperatures of $\mathrm{TMZ}$ and $\beta-\mathrm{CD}$ appear at the exact

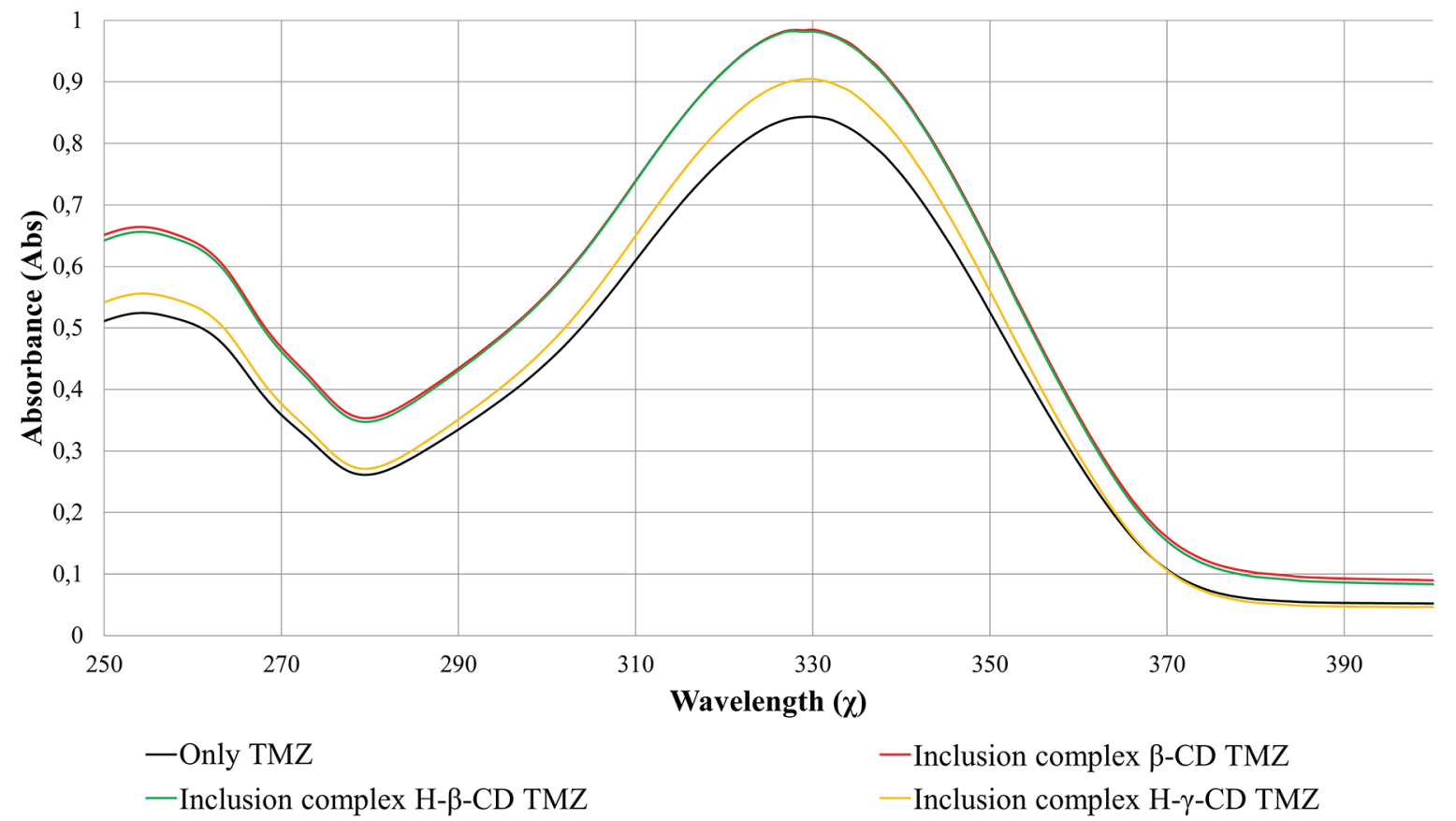

FIGURE 8 - Amount of solubilized TMZ in $\mathrm{mg} / \mathrm{mL}$ in $0.1 \mathrm{M} \mathrm{HCl}$ in the presence of different CDs in 1:1 ratio after the formation of inclusion complex after 24 hours (UV-Vis results). 
TABLE II - Amount of solubilized TMZ in $\mathrm{mg} / \mathrm{mL}$ in $0.1 \mathrm{M} \mathrm{HCl}$ after the formation of inclusion complex after one hour and 24 hours

\begin{tabular}{lcccccc}
\hline \multirow{2}{*}{ Formulations } & \multicolumn{2}{c}{$\begin{array}{c}\text { 1 mol TMZ: 1 mol CD } \\
(\mathbf{m g} / \mathbf{m L})\end{array}$} & \multicolumn{2}{c}{$\begin{array}{c}\text { 1 mol TMZ: 2 mol CD } \\
(\mathbf{m g} / \mathbf{m L})\end{array}$} & \multicolumn{2}{c}{$\begin{array}{c}\text { 1 mol TMZ:5 mol CD } \\
(\mathbf{m g} / \mathbf{m L})\end{array}$} \\
\cline { 2 - 7 } & One hour & $\mathbf{2 4}$ hours & One hour & $\mathbf{2 4}$ hours & One hour & $\mathbf{2 4}$ hours \\
\hline Only TMZ & & \multicolumn{2}{c}{3.46} \\
$\beta-C D-T M Z$ & $4.23 \pm 0.16$ & $4.23 \pm 0.40$ & $4.43 \pm 0.14$ & $4.31 \pm 0.49$ & $4.59 \pm 0.07$ & $4.51 \pm 0.07$ \\
Hydroxy- $\beta$-CD-TMZ & $4.13 \pm 0.14$ & $4.29 \pm 0.04$ & $4.3 \pm 0.09$ & $4.26 \pm 0.4$ & $4.43 \pm 0.4$ & $4.25 \pm 0.1$ \\
Hydroxy- $\gamma$-CD-TMZ & $4.33 \pm 0.16$ & $4.29 \pm 0.04$ & $4.35 \pm 0.07$ & $4.43 \pm 0.02$ & $4.53 \pm 0.5$ & $4.52 \pm 0.8$ \\
\hline
\end{tabular}

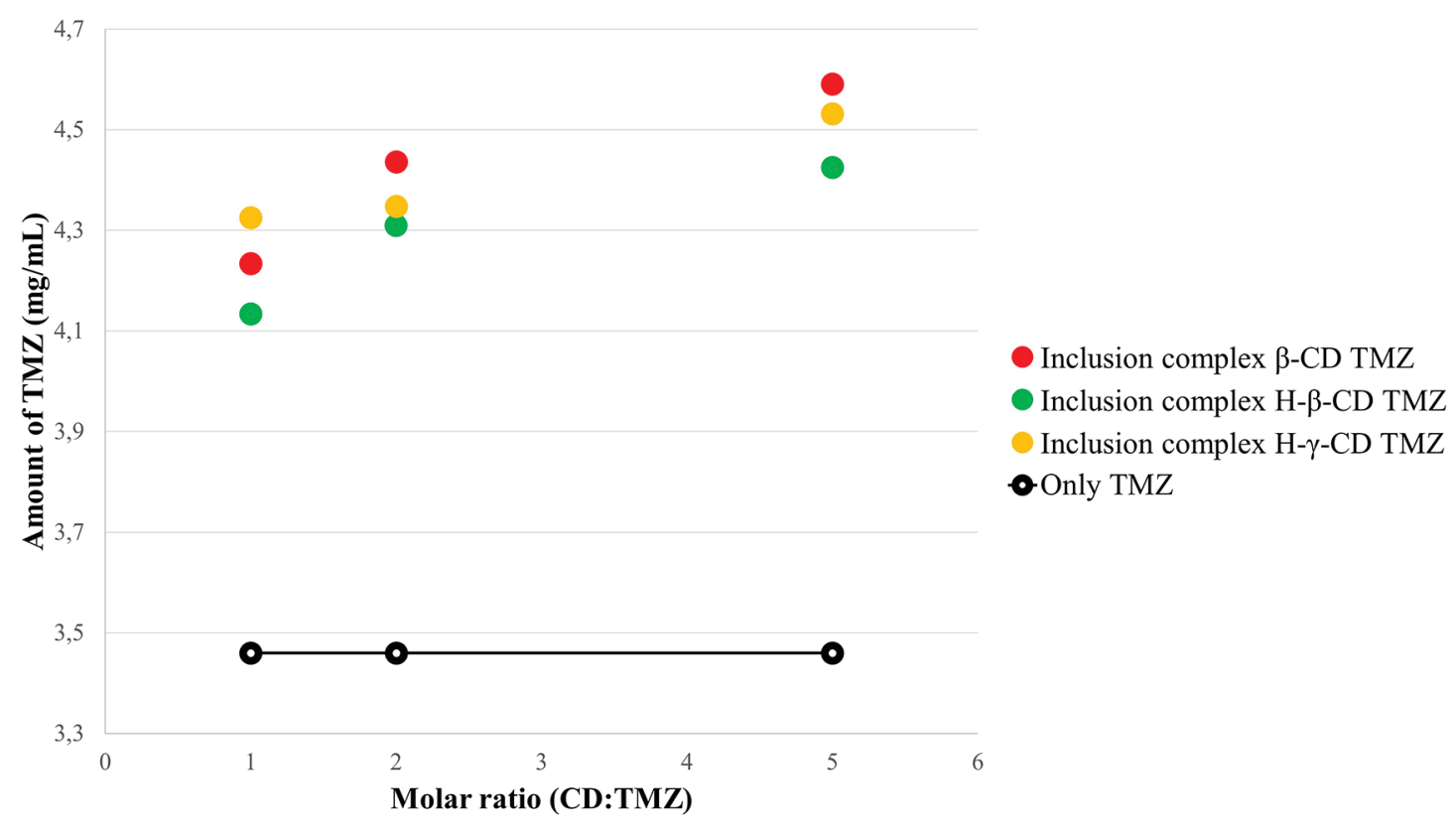

FIGURE 9 - Amount of solubilized TMZ in $\mathrm{mg} / \mathrm{mL}$ in $0.1 \mathrm{M} \mathrm{HCl}$ after the formation of inclusion complex after one hour.

temperatures. On the other hand, in the complex, two baseline temperatures of TMZ and $\beta$-CD were observed, but with temperature shifts of 7 and $15{ }^{\circ} \mathrm{C}$, respectively, which was quite significant, indicating the interaction of TMZ and $\beta-C D$ at the complexation level. Baseline temperatures are often used in the verification of a compound (Widmann, 2000). Shifts in the baseline temperatures and new weight loss profiles upon complex formation have been obtained by other researchers (Banerjee, Chen, 2007) and therefore used as an additional support for the formation of an inclusion complex between TMZ and $\beta$-CD. DSC (Figure 7) results can also be interpreted in a similar fashion where the physical mixture exhibits similar heat profiles to its pure constituents, and the complex has a totally different heat profile in the same temperature region, indicating the formation of a new complex. Somewhat similar endothermic dehydration is still observed, expected from the water being lost from $\beta$-CD. However, the exothermic decomposition peak of TMZ completely disappears from its relevant position. In the literature, DSC profiles of cyclodextrin inclusion complexes with salbutamol (Marques, Hadgraft, Kellaway, 1990) and quercetin (Pralhad, Rajendrakumar, 2004) have been investigated and were shown to possess their unique heat loss profile, significantly exempted from the exothermic decomposition peak of the drug, showing that the drug participates in the inclusion complex. All the methods employed for the confirmation of the formation of an inclusion complex show that a complex has indeed formed between TMZ and CDs, and its study properties are significantly different from that of the physical mixture. The solubility of temozolomide in water was determined using UV absorbance as shown in Figure 4 to be 3.46 $\mathrm{mg} / \mathrm{mL}$. In literature, this value is stated as $5.09 \mathrm{mg} / \mathrm{mL}$ based on the ALOGPS calculations (http://www.vcclab. org) However, it is expected that experimental values may not correspond exactly with the calculated values. The solubility values obtained from UV are consistent 
with HPLC results reported in Table I, both indicating a solubility of around $3.5 \mathrm{mg} / \mathrm{mL}$ at $25^{\circ} \mathrm{C}$. Once this value was found, solubility enhancement experiments were conducted. These experiments have shown that in the presence of cyclodextrins, the solubility of the drug increases by about 25\%, as clearly shown in Figure 9 where the black line indicates the solubility of TMZ whereas the other data is of different complexes. One important finding is that the drug does not appear to have a significant preference towards either of the cyclodextrins, indicating that the cavity size is large enough for even the smallest sized ones ( $\beta-\mathrm{CD}$ and $\mathrm{H}-\gamma-\mathrm{CD})$. Upon increasing the $\mathrm{CD}$ amount in the medium, the solubility of TMZ is further increased, but not in a linear fashion. This may suggest that there may be more than the simple one-to-one complexation mechanism taking place when the $\mathrm{CD}$ concentration is increased. It is likely that in the presence of high amounts of CD, one TMZ molecule may be complexing with more than one $\mathrm{CD}$ molecule, resulting in this nonlinear enhancement of solubility with increasing $\mathrm{CD}$ concentration. In literature, although there are some reports indicating a clear preference of the drug towards one of the CDs (Yoshida et al., 1989) or a linear increase in the solubility with increasing concentration (type A solubility), similar results to our findings have also been reported (type B solubility), where various types are described by Higuchi and Connors (1965). All these findings indicate the formation of inclusion complexes between temozolomide and different CDs, and these inclusion complexes enhancing the solubility of TMZ, which is very low in aqueous medium, by about $25 \%$.

\section{CONCLUSION}

Temozolomide, an anti-cancer drug, which is quite unstable and insoluble in aqueous medium, is nearly impossible to be targeted in the body due to its severe side effects. In order to increase the solubility of this drug different cyclodextrins ( $\beta$-CD, 2-hydroxy-propyl$\beta$-CD, 2-hydroxy-propyl- $\gamma$-CD) have been used, and the drug is shown to form an inclusion complex with these cyclodextrins by using SEM, TGA, DSC and HPLC. As a result of this complex formation, it has been shown that drug solubility is increased in the presence of cyclodextrins, regardless of the type cyclodextrin. Increasing the amount of cyclodextrin increases solubility in the complex, although not in a direct proportion. All experiments have been carried out in the acidic media to prevent hydrolysis of TMZ to its active agent, MTIC at neutral $\mathrm{pH}$. Once solubility is enhanced, the drug-CD inclusion complex can be freeze-dried to be filled in a capsule, as it is formulated in the market. This study shows that cyclodextrins can be used as complexing agents for poorly soluble anti-cancer drugs, increasing the solubility and hence the availability of the drug.

\section{ACKNOWLEDGEMENT}

We would like to give special thanks to Dr. Mustafa Ilhan at Marmara University for the SEM images. This project is funded by TUBITAK, with Project number $113 \mathrm{M} 265$

\section{REFERENCES}

Aiassa V, Zoppi A, Albesa I, Longhi MR. Inclusion complexes of chloramphenicol with $\beta$-cyclodextrin and aminoacids as a way to increase drug solubility and modulate ROS production. Carbohydr Polym. 2015;121:320-327.

Andrasi M, Bustos R, Gaspar A, Gomez FA., Klekner A. Analysis and stability study of temozolomide using capillary electrophoresis. J Chromatogr B Biomed Sci Appl. 2010;878(21):1801-1808.

Atipairin A, Sawatdee S. Inclusion complexes between sildenafil citrate and cyclodextrins enhance drug solubility. Asian J Pharm Sci. 2016;11:104-105.

Babu NJ, Sanphui P, Nangia A. Crystal engineering of stable temozolomide cocrystals. Chem Asian J. 2012;7(10):2274-2285.

Banerjee SS, Chen DH. Magnetic nanoparticles grafted with cyclodextrin for hydrophobic drug delivery. Chem Mater. 2007;19(25):6345-6349.

Brada M, Judson I, Beale P, Moore S, Reidenberg P, Statkevich $\mathrm{P}$, et al. Phase I dose-escalation and pharmacokinetic study of temozolomide (SCH 52365) for refractory or relapsing malignancies. Br J Cancer. 1999;81(6):1022-1030.

Chidambaram M, Manavalan R, Kathiresan K. Nanotherapeutics to overcome conventional cancer chemotherapy limitations. J Pharm Pharm Sci. 2011;14(1):67-77.

De Araujo, DR Tsuneda, SS Cereda, CM Del Carvalho, F, Preté, PS, Fernandes, et al. Development and pharmacological evaluation of ropivacaine-2-hydroxypropyl- $\beta$-cyclodextrin inclusion complex. Eur J Pharm Sci. 2008;33(1):60-71.

Del Valle EMM. Cyclodextrins and their uses: a review. Process Biochem. 2004;39(9):1033-1046. 
Franco C, Schwingel L, Lula I, Sinisterra RD, Koester LS, Bassani VL. Studies on coumestrol/ $\beta$-cyclodextrin association: Inclusion complex characterization. Int J Pharm. 2009;369(12):5-11.

Freitas MR, Rolim LA, Soares MFLR., Rolim-Neto PJ, Albuquerque MM, Soares-Sobrinho JL. Inclusion complex of methyl- $\beta$-cyclodextrin and olanzapine as potential drug delivery system for schizophrenia. Carbohydr Polym. 2012;89(4):10951100 .

Hamada H, Ishihara K, Masuoka N, Mikuni K, Nakajima N. Enhancement of water-solubility and bioactivity of paclitaxel using modified cyclodextrins. J Biosci Bioeng. 2006;102(4):369-371.

Higuchi T, Connors A. Phase-solubility techniques. Adv Chem Inst. 1965;4:212-217.

Khan A, Imam SS, Aqil M, Ahad A, Sultana Y, Ali A, Khan $\mathrm{K}$. Brain targeting of temozolomide via the intranasal route using lipid-based nanoparticles: brain pharmacokinetic and scintigraphic analyses. Mol Pharm. 2016;13(11):3773-3782.

Kicuntod J, Khuntawee W, Wolschann P, Pongsawasdi P, Chavasiri W, Kungwan N, Rungrotmongkol T. Inclusion complexation of pinostrobin with various cyclodextrin derivatives. J Mol Graph Model. 2016;63:91-8.

Kim H, Likhari P, Parker D, Statkevich P, Marco A, Lin CC, et al. High-performance liquid chromatographic analysis and stability of anti-tumor agent temozolomide in human plasma. $\mathrm{J}$ Pharm Biomed Anal. 2001,24(3):461-468.

Loftsson T, Magnusdottir A, Masson M, Sigurjonsdottir JF. Selfassociation and cyclodextrin solubilization of drugs. J Pharm Sci. 2002;91(11):2307-2316.

Loftsson T, Jarho P, Másson M, Järvinen T. Cyclodextrins in drug delivery. Expert Opin Drug Deliv. 2005;2(2):335-351.

Maestrelli F, González-Rodríguez ML, Rabasco AM, Ghelardini C, Mura P. New "drug-in cyclodextrin-in deformable liposomes" formulations to improve the therapeutic efficacy of local anaesthetics. Int J Pharm.2010;395(1-2):222-231.

Marchesi F, Turriziani M, Tortorelli G, Avvisati G, Torino F, De Vecchis L. Triazene compounds: mechanism of action and related DNA repair systems. Pharmacol Res. 2007;56(4):275-87.
Marques HC, Hadgraft J, Kellaway I. Studies of cyclodextrin inclusion complexes. I. The salbutamol-cyclodextrin complex as studied by phase solubility and DSC. Int J Pharm. 1990;63(3):259-266.

Ol'khovich MV, Sharapova AV, Lavrenov SN, Blokhina SV, Perlovich, GL. Inclusion complexes of hydroxypropyl- $\beta$ cyclodextrin with novel cytotoxic compounds: Solubility and thermodynamic properties. Fluid Phase Equilib. 2014;384:6872 .

Payne MJ, Pratap SE, Middleton MR. Temozolomide in the treatment of solid tumours: current results and rationale for dosing/scheduling. Crit Rev Oncol Hematol. 2005;53(3):241252 .

Pralhad T, Rajendrakumar K. Study of freeze-dried quercetincyclodextrin binary systems by DSC, FT-IR, X-ray diffraction and SEM analysis. J Pharm Biomed Anal. 2004;34(2):333-339.

Priya AS, Sivakamavalli J, Vaseeharan B, Stalin T. Improvement on dissolution rate of inclusion complex of Rifabutin drug with $\beta$-cyclodextrin. Int J Biol Macromol. 2013;62:472-480.

Ruan LP, Yu BY, Fu GM, Zhu DN. Improving the solubility of ampelopsin by solid dispersions and inclusion complexes. J Pharm Biomed Anal. 2005;38(3):457-464.

Shende PK, Gaud RS, Bakal R, Patil D. Effect of inclusion complexation of meloxicam with $\beta$-cyclodextrin- and $\beta$-cyclodextrin-based nanosponges on solubility, in vitro release and stability studies. Colloids Surf B Biointerfaces. 2015;136:105-110.

Stupp R, Mason WP, Van Den Bent MJ, Weller M, Fisher B, Taphoorn MJ, et al. Radiotherapy plus concomitant and adjuvant temozolomide for glioblastoma. N Eng J Med.2005;352(10):987-96.

Tang P, Li S, Wang L, Yang H, Yan J, Li H. Inclusion complexes of chlorzoxazone with $\beta$ and hydroxypropyl- $\beta$ cyclodextrin:characterization, dissolution, and cytotoxicity. Carbohydr Polym. 2015;131:297-305.

Trotta F, Zanetti M, Camino G. Thermal degradation of cyclodextrins. Polym Degrad Stab. 2000;69:373-379.

Uekama K, Otagiri M. Cyclodextrins in drug carrier systems. Crit Rev Ther Drug Carrier Syst.1987;3(1):1-40. 
VCCLAB. Virtual Computational Chemistry Laboratory, 2005. Available from: http://www.vcclab.org.

Widmann G. Interpreting TGA curves, 2000. [cited 2001 Jan 1]. Available from: http://www.masontechnology.ie/x/Usercom_13. pdf.

Xiao CF, Li K, Huang R, He GJ, Zhang JQ, Zhu L, et al. Investigation of inclusion complex of Epothilone A with cyclodextrins. Carbohydr Polym. 2014;102:297-305.

Yoshida A, Yamamoto M, Irıe T, Hırayama F, Uekama K. Some pharmaceutical properties of 3-hydroxypropyl-and 2, 3 -dihydroxypropyl- $\beta$-cyclodextrins and their solubilizing and stabilizing abilities. Chem Pharm Bull. 1989;37(4):1059-1063.

Zhang W, Gong X, Cai Y, Zhang C, Yu X, Fan J. Investigation of water-soluble inclusion complex of hypericin with betacyclodextrin polymer. Carbohydr Polym. 2013a;95(1):366-370.
Zhang Y, Ren K, He Z, Li H, Chen T, Lei Y, Development of inclusion complex of brinzolamide with hydroxypropyl- $\beta$ cyclodextrin. Carbohydr Polym. 2013b;98(1):638-643.

Zhang D, Zhang J, Jiang K, Li K, Cong, Y, Pu S, et al. Preparation, characterisation and antitumour activity of $\beta$-, $\gamma$ - and HP- $\beta$-cyclodextrin inclusion complexes of oxaliplatin. Spectrochim Acta A: Mol Biomol Spectrosc. 2016;152:501-508.

Zuckerman JE, Hsueh T, Koya RC, Davis ME, Ribas A. siRNA knockdown of ribonucleotide reductase inhibits melanoma cell line proliferation alone or synergistically with temozolomide. J Invest Dermatol. 2011,131(2):453-460.

Received for publication on $24^{\text {th }}$ August 2017 Accepted for publication on $14^{\text {th }}$ November 2017 